\title{
Research on Health Node Capture Method under Computer Network Intrusion
}

\author{
Xu Guibing, Zhou Xuemin \\ Nanchang Institute of Technology, Nanchang, Jiangxi, 330044, China
}

Keywords: Computer network, Health node, Ahp, Three side centroid localization

\begin{abstract}
It is of great significance to ensure the safe operation of the network by identifying the location of the health node quickly and accurately and taking appropriate measures to protect the computer network. In view of the disadvantages of traditional node capture method with high positioning error and long running time, a new method based on three edge centroid positioning principle is proposed to capture health nodes. According to the analytic hierarchy process, the importance weight ratio of the node signal output array, the node number, the node degree, the eigenvector and the node density is determined, and the approximate location of the existing health nodes in the computer network is identified, and the anchor node and the selected unknown node are determined based on the three edge centroid localization method. The optimal distance between nodes and healthy nodes can accurately locate and capture healthy nodes in computer networks. The experimental data show that the proposed health node capture method can get lower positioning error under the condition of different node density and signal to noise ratio, and also have some advantages in node capture energy consumption control and operation time control.
\end{abstract}

\section{Introduction}

The rapid development of computer network technology has brought great convenience to people's study, work and life, but at the same time, network intrusion has become more and more rampant. Network intrusion not only threatens the key data and privacy of network users, but also faces the risk of paralysis once most nodes are attacked. After the computer network is attacked by intrusion, the uninfected key health nodes can also communicate with each other, which can keep the network stable and unblocked temporarily. Therefore, in the case of computer network intrusion, how to identify the existing healthy nodes in the network in a short time, and accurately capture and locate these healthy nodes, has become one of the key measures to protect the temporary security of the network. For the analysis and research of the existing computer network health node capture technology, some scholars put forward the method of computer network health node capture based on particle swarm optimization algorithm, which can realize the accurate positioning of nodes, but the cost of this method is too high; and a health node capture method based on distributed Alamouti coding is designed, which is relatively simple in the process of positioning, but overall In addition, some scholars put forward a random bandwidth weighted method to identify and capture healthy nodes, but the communication efficiency of this method is too low to meet the basic communication requirements of computer network under malicious data attack. In view of the shortcomings of traditional methods of capturing healthy nodes in computer networks, a new method of locating and capturing healthy nodes in computer networks based on the theory of three-sided mass center is proposed. Based on the traditional anchor node positioning principle, this method introduces the concept of hierarchical analysis, and first identifies the general area of the health node based on the judgment of indicators, so as to reduce the time of health positioning. Then through the selection and determination of the optimal distance among anchor nodes, unknown nodes and healthy nodes, the accurate capture of healthy nodes in the network is completed. The simulation experimental data also verify the superiority and effectiveness of the proposed method.

\section{Location Identification of Healthy Nodes in Computer Network}

In order to accurately locate and capture healthy nodes in a computer network, and ensure the 
smoothness of information transmission after the network is attacked, it is necessary to first identify the approximate locations of key healthy nodes based on the relevant topology parameters and evaluation indicators in the computer network. In order to shorten the time consumption of healthy node positioning. The communication characteristics of healthy nodes and attacked abnormal nodes are different, and the non-linear sequences of signals in the information transmission process are also different. Let the communication signal sources of healthy nodes in the computer network be $\tau_{1}(t), \tau_{2}(t), \ldots, \tau_{n}(t)$, The distance of the output array of node signals in the network topology at this time $d$,The following conditions are met:

$$
d \leq 2 \frac{r^{2} \tau(t)}{\lambda}
$$

Where, $r$ is the communication radius of the node , $\lambda$ is the wavelength of the communication signal. The output array and related topology parameters of healthy nodes in computer network are the basic conditions to study node capture. The general location of healthy nodes in the network will be affected by a number of factors, including node degree, node eigenvector, the number of healthy nodes and node compactness. Among them, the node degree index $\eta(i)$ is one of the important indexes to judge the importance of nodes in the computer network and to determine the location of healthy nodes. Node in computer network $i$ in a computer network can be defined as the number of edges of all nodes adjacent to the node $i$, which is represented $k_{i}$ by parameters. If the index $i$ value of the node $k_{i}$ is larger, it is proved that the node is more important. In the rescue of the health nodes in the attacked network, we need to take corresponding protection measures for these important nodes first.

If the node $p$ is the neighbor of the node $i$, the number of nodes $p$ passing through the node $\xi(p)$ is the ratio of the optimal path passing through the node $p$ to the optimal path $\kappa_{i j}$ in the whole topological network:

$$
\xi(p)=\sum_{i, j}^{n} \frac{\kappa_{i j}(p)}{\kappa_{i j}}
$$

Among them, $i$ and $j$ are the nodes adjacent to the nodes $p$ in the complex computer network, $n$ are the number of nodes in the whole network. The eigenvector of a node refers to the maximum eigenvalue of the eigenmatrix adjacent to the node $i$, which is one of the main indicators to determine the exact area where the node is located and the health level of the node. The eigenvector of a healthy node $\varsigma(i)$ can be expressed as:

$$
\varsigma(i)=\frac{1}{\lambda} \sum_{i, j=1}^{n} \alpha_{i j} e_{j}
$$

Among them, $\lambda$ it represents the main eigenvalue between adjacent matrices in a complex computer network, which $e_{j}=\left\{\begin{array}{ll}e_{1}, e_{2}, \ldots . ., e_{j}\end{array}\right\}^{T}$ is the eigenvector. Parameters $\alpha_{i j}$ can determine whether there is edge connection between nodes.

The compactness index $\zeta(i, j)$ between nodes $i$ represents the sum of the shortest communication distance with all nodes adjacent to the nodes, which is expressed as:

$$
\zeta(i, j)=\sum_{i, j=1}^{n} d_{i j}
$$

For the determination and recognition of the general location of healthy nodes in complex computer networks, five index factors, such as the output array of node signal, node degree, node 
eigenvector, the number of healthy nodes and node compactness, will play a very important role. It is the key to identify healthy nodes to accurately analyze the proportion of different influence indexes. Based on the analytic hierarchy process, this paper determines the weight proportion of each index, and finally solves the importance value of health node. The health degree and importance degree of the nodes in the computer network depend on the above five index factors. If the index weights of these influencing factors are $w_{1}, w_{2}, w_{3}, w_{4}, w_{5}$, the health degree indexes of the nodes IMP in the computer network can be expressed as:

$$
\begin{aligned}
& I M P=w_{1} \times \tau(i)+w_{2} \times \eta(i)+w_{3} \times \xi(p) \\
& w_{4} \times \varsigma(i)+w_{5} \times \zeta(i, j)
\end{aligned}
$$

Based on the index IMP and AHP, the hierarchy analysis structure and the judgment matrix of node health degree are constructed, and the recognition level of health nodes in the computer network is shown in Figure 1:

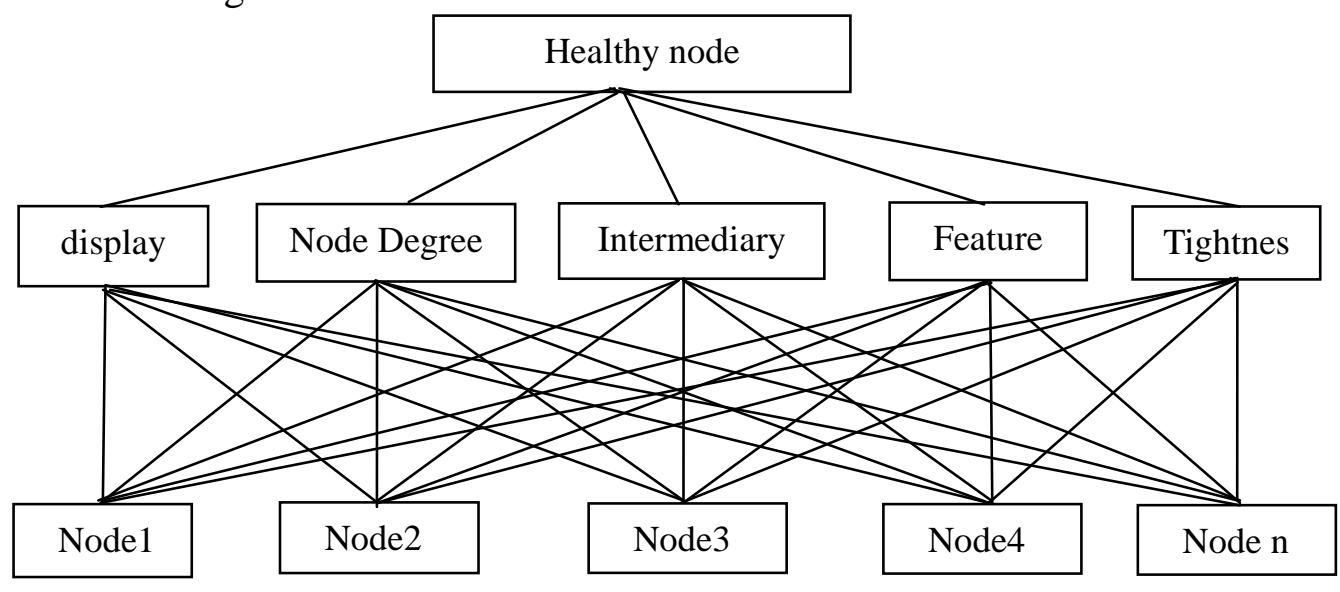

Fig.1 Hierarchy of Health Node Identification in Computer Network

The consistency of the matrix is tested by eigenvector method, and then the radius area of the key health nodes in the computer network is determined based on the index value. Finally, the accurate location and capture of the health nodes are realized through the determination of the accurate location of the nodes.

\section{The Realization of Health Node Location Capture Based on Three Edge Centroid Location Algorithm}

Based on the analytic hierarchy process (AHP) theory, the key health nodes in the computer network are identified, and the communication radius area of the nodes can be determined. Based on the three edge centroid localization algorithm to achieve the accurate capture and location of healthy nodes, we need to use the anchor nodes that have been accurately located in the radius area of healthy nodes, set the three anchor nodes within the communication radius of the healthy nodes $P$ to be captured in the computer network as $A\left(x_{1}, y_{1}\right), B\left(x_{2}, y_{2}\right), C\left(x_{3}, y_{3}\right)$, and the test distance from the anchor nodes to the healthy nodes $P$ as $d_{1}, d_{2}$ and $d_{3}$.

Definition 1: in order to improve the accuracy of $n$ health node capture, the unknown nodes $\left(x_{4}, y_{4}\right)$, and $\left(x_{5}, y_{5}\right), \ldots,\left(x_{n+3}, y_{n+3}\right)$, the distance between these unknown nodes and health nodes $P$ is $d_{4}, d_{5}, \ldots, d_{n+3}$. According to the above definition, the sum of the distances between the healthy nodes $\left(x_{i}, y_{i}\right)$ and the nodes $\left(x_{j}, y_{j}\right)$ can be calculated. In the solution, the point coordinates which are closest to the health nodes $P$ are obtained $\left(x_{k}, y_{k}\right)$. By comparing the measurement distance, we can find the near point of space location $\left(x_{i}, y_{i}\right),\left(x_{j}, y_{j}\right)$ which is close 
to the health node.

Theorem 1: for the best distance point $\left(x_{k}, y_{k}\right)$, there are three situations: there is a sum of two points $\left(x_{i}, y_{i}\right)$ and $\left(x_{j}, y_{j}\right)$, a point $\left(x_{i}, y_{i}\right)$ or $\left(x_{j}, y_{j}\right)$, or there is no such point. When the solution does not exist, there is no point in the space, and only the anchor node positioning method can be used; when the solution is one point, the point $i$ or point $j$ can be used as the positioning point, and the three anchor nodes can jointly realize the positioning of the healthy node; when the solution is two points, set the solution $\left(x^{\prime}, y^{\prime}\right)$ and $\left(x^{\prime \prime}, y^{\prime \prime}\right)$ as sum, and the solution closer to the optimal distance point $\left(x_{k}, y_{k}\right)$ is the optimal solution.

Based on the above definitions and theorems, select the unknown nodes $\left(x_{q 1}, y_{q 1}\right)$ and $\left(x_{q 2}, y_{q 2}\right) \mathrm{t}$, find the best location near point relative to the healthy nodes $P$ :

$$
\left\{\begin{array}{l}
\sqrt{\left(x-x_{q 1}\right)^{2}+\left(y-y_{q 1}\right)^{2}}=d_{q 1} \\
\sqrt{\left(x-x_{q 2}\right)^{2}+\left(y-y_{q 2}\right)^{2}}=d_{q 2}
\end{array}\right.
$$

Calculate the distance from the two selected unknown nodes to the anchor nodes $A\left(x_{1}, y_{1}\right), B\left(x_{2}, y_{2}\right), C\left(x_{3}, y_{3}\right)$ :

$$
\begin{aligned}
& \left\{\begin{array}{l}
\sqrt{\left(x_{1}-x_{q 1}\right)^{2}+\left(y_{1}-y_{q 1}\right)^{2}}=d_{1 a} \\
\sqrt{\left(x_{1}-x_{q 2}\right)^{2}+\left(y_{1}-y_{q 2}\right)^{2}}=d_{1 b}
\end{array}\right. \\
& \left\{\begin{array}{l}
\sqrt{\left(x_{2}-x_{q 1}\right)^{2}+\left(y_{2}-y_{q 1}\right)^{2}}=d_{2 a} \\
\sqrt{\left(x_{2}-x_{q 2}\right)^{2}+\left(y_{2}-y_{q 2}\right)^{2}}=d_{2 b}
\end{array}\right. \\
& \left\{\begin{array}{l}
\sqrt{\left(x_{3}-x_{q 1}\right)^{2}+\left(y_{3}-y_{q 1}\right)^{2}}=d_{3 a} \\
\sqrt{\left(x_{3}-x_{q 2}\right)^{2}+\left(y_{3}-y_{q 2}\right)^{2}}=d_{3 b}
\end{array}\right.
\end{aligned}
$$

Then the distance between the near point and the far point is compared by using the difference value

$$
\left\{\begin{array}{l}
\left|d_{1}-d_{q 1}\right| \stackrel{?}{\geq}\left|d_{1}-d_{q 2}\right| \\
\left|d_{2}-d_{q 1}\right| \stackrel{?}{\geq}\left|d_{2}-d_{q 2}\right| \\
\left|d_{3}-d_{q 1}\right| \stackrel{?}{\geq}\left|d_{3}-d_{q 2}\right|
\end{array}\right.
$$

When comparing $\left|d_{i}-d_{q 1}\right|$ with $\left|d_{i}-d_{q 2}\right|$, it represents the distance between the first node and the health node. If the left side is larger than the right side, you can select the node $Q_{1}$ and the three anchor nodes to accurately capture the location of the health node, and delete the relevant data of the node $Q_{2}$; if the left side is smaller than the right side, you can select the node $Q_{2}$ and the three anchor nodes to accurately capture the location of the health node, and delete the relevant number of nodes $Q_{1}$.According to. Based on the original three anchor nodes, the three edge centroid 
localization algorithm increases the participation of two spatial positioning nodes, improves the capture accuracy of healthy nodes in the computer network, and reduces the number of sampling.

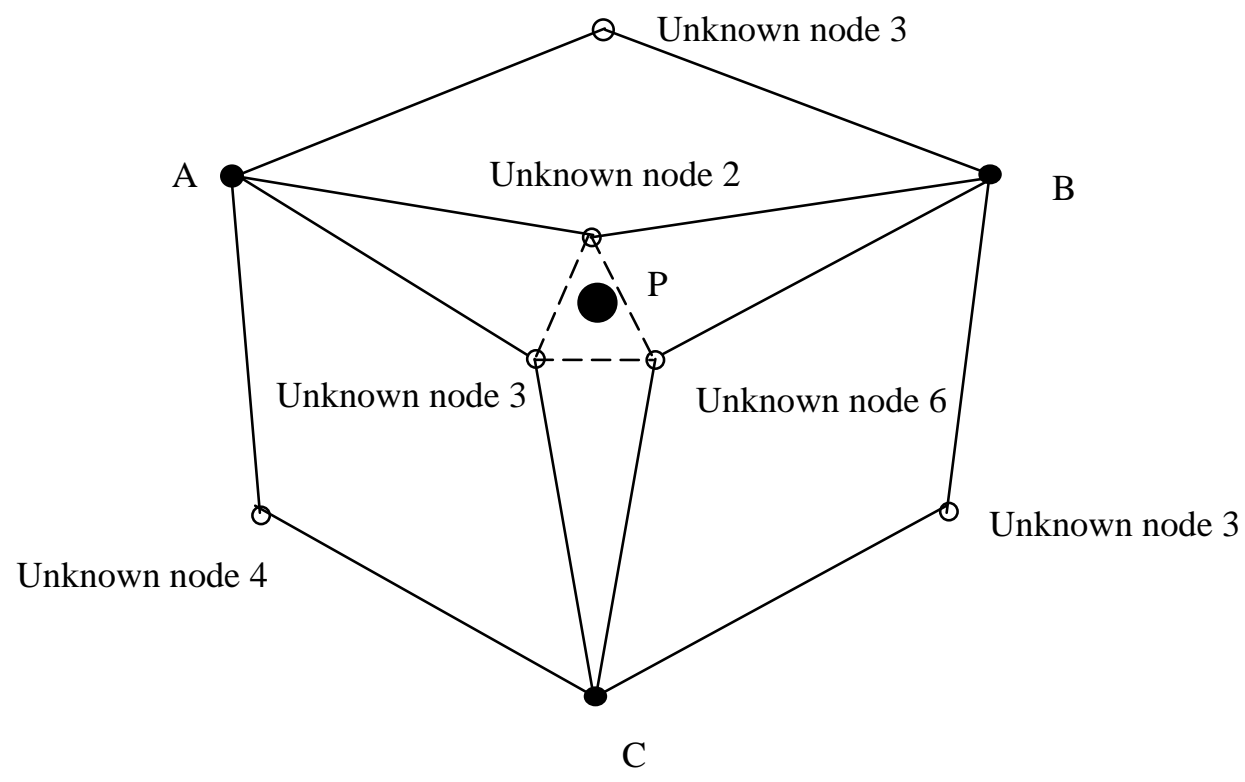

Fig.2 Three Edge Centroid Algorithm Model

Based on the analytic hierarchy process (AHP), this paper determines the factors that affect the location of health nodes. Based on the traditional anchor node location theory, two unknown nodes are introduced to participate in the capture and location of health nodes. Based on the determination of the optimal distance between anchor nodes, unknown nodes and health nodes, the existing health nodes in the computer network are accurately captured.

\section{Experimental Part}

In order to determine the application performance of the computer network health node capture method proposed in the paper, the simulation experiment is carried out based on the matlab simulation tool, the computer network model is established in the area of 500*500m, 200 nodes are evenly distributed, and other relevant computer network parameters are set, as shown in Table 1:

Table 1 Parameters Setting Of Computer Network Health Node Capture

\begin{tabular}{|l|l|l|l|}
\hline Network node & Parameter value & Signal frequency & Parameter value \\
\hline Number of nodes & 200 & Frequency band & $4-20 \mathrm{KHz}$ \\
\hline Communication radius & $50 \mathrm{~m}$ & Initial frequency & $0.5 \mathrm{KHz}$ \\
\hline Network SNR & $2-60 \mathrm{~dB}$ & Middle finger frequency & $0.8 \mathrm{KHz}$ \\
\hline Node density & 30 & Positioning scan & $7.5 \mathrm{KHz}$ \\
\hline Number of anchor nodes & 25 & - & - \\
\hline Positioning cycle & $50 \mathrm{~s}$ & - & - \\
\hline
\end{tabular}

\subsection{Capture and Positioning Error of Healthy Nodes}

The uninfected healthy nodes are located in the known computer network structure. The location accuracy and error level will be affected by the number of nodes and the network signal-to-noise ratio and other factors. Therefore, under different parameter settings, the performance of the health node capture method and the particle group location capture method in the paper is compared and verified. The data statistical analysis results are shown in Figure 3 and figure 3 Figure 4 shows: 


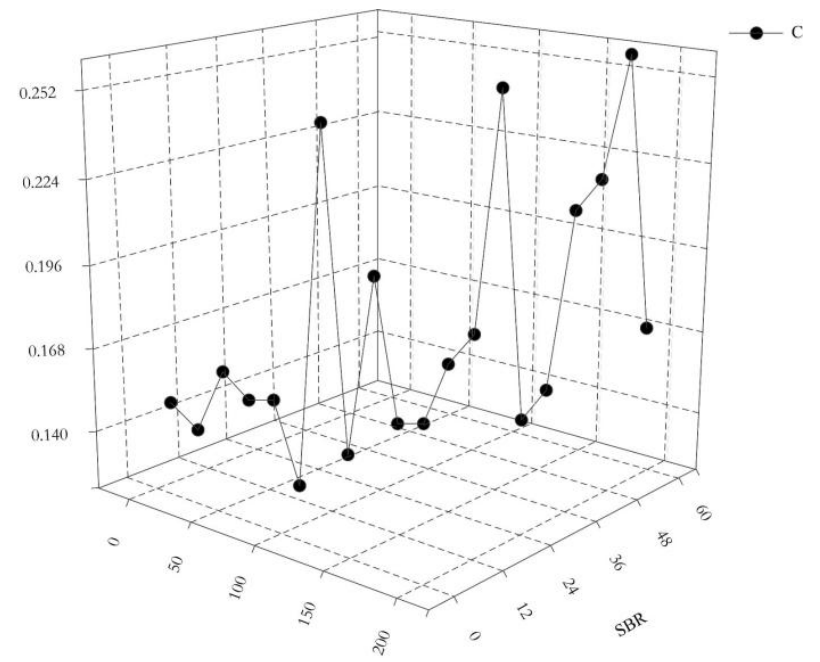

Fig.3 Positioning Error of Health Node Capture Method in the Paper

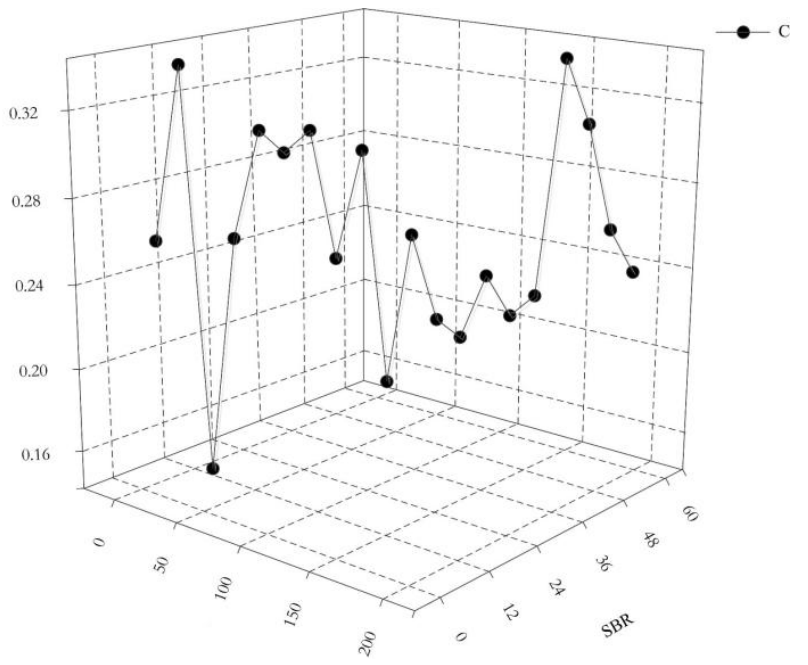

Fig.4 Positioning Error of Health Node Capture Method

From the analysis of the maximum error values of the two health node capture methods in Figure 3 and Figure 4, in this paper, the maximum error of the node capture method based on the three side centroid principle is not more than $0.25 \%$, and most of the error data points are controlled below $0.2 \%$; while the error control rate of the health node capture and location method based on particle swarm is poor, and most of the positioning errors of the data points are higher than $0.2 \%$, and the fluctuation is large . insufficient stability.

\subsection{Comparison of Energy Consumption of Health Node Capture and Location}

The energy of the nodes in the computer network is supplied by the battery. Because the energy of the nodes is limited, the impact of repeated sampling positioning on the energy consumption of the nodes should be considered in the capture and positioning of healthy nodes, so as to reduce the number of unnecessary sampling positioning as much as possible, save the energy consumption of nodes and ensure the communication stability of the computer network. Under different node density, firstly, the healthy node capture method is proposed for sampling location. Under the condition of sampling node density of 40-120, three kinds of healthy nodes capture sampling times. Under different node density conditions, the sampling times of the method in this paper are the lowest, so it can save the energy loss caused by node location objectively. 


\subsection{Comparison of Method Operation Time}

After the computer network is attacked, the network virus or malicious data will attack the only remaining healthy nodes at a very fast speed, so the capture and location time of healthy nodes is very important to maintain the stability of the network, which is also one of the important indicators to measure the performance of the method. From the analysis of the capture and location time of the healthy nodes, the method in this paper has advantages over the traditional method in terms of the overall operation time or the operation time of a single node, thus verifying the practical application efficiency of the method based on the three edge centroid location.

\section{Conclusion}

Under the condition of network intrusion, malicious data will spread among nodes. The capture, location and protection of healthy nodes can win valuable time for network rescue. This paper proposes a method of capturing healthy nodes based on the principle of trilateral centroid positioning, which first identifies the location of healthy nodes, and then accurately captures the location in real time, which improves the positioning accuracy of healthy nodes, and reduces the energy consumption of node positioning.

Project: Twelfth Five-Year Plan Project "Application Research on Student Associations to Promote the Cultivation of Innovative Talents in Private Colleges and Universities”

\section{References}

[1] Sun Lei.(2019).Intrusion and defense technology of computer network server [J]. Electronic technology and software engineering, no.24,pp.182-183.

[2] Liu yubiao.(2019).Application of artificial intelligence technology in computer network intrusion detection [J]. Science and technology wind, no.32,pp.94 + 97.

[3] Xiong Ganjin.(2019).The construction of the optimal path deduction model of ship computer network intrusion [J]. Ship science and technology, vol.41,no.18,pp.148-150.

[4] Chen Changqing.(2019).Research on intrusion prevention method of computer network communication based on data encryption technology [J]. Information and computer (theoretical Edition), no.14,pp.171-172.

[5] Gao Wei.(2018).Application of artificial intelligence technology in computer network technology [J]. Journal of Liaoning University of science and technology, vol.20,no.2,pp.1-3 + 9.

[6] Hu Yan.(2017).Research and Simulation of communication selection algorithm for healthy nodes in computer network intrusion environment [J]. Microelectronics and computer, vol.34, no.1,pp. 141-144. 